\title{
The Antioxidant Resveratrol Protects against Chondrocyte Apoptosis by Regulating the COX-2/NF- $\kappa$ B Pathway in Created Temporomandibular Osteoarthritis
}

\author{
Wen Li $\mathbb{D}^{D}$, Shiyu Hu $\left(\mathbb{D}\right.$, Xuepeng Chen $\mathbb{D}$, and Jiejun Shi $\mathbb{C}^{\circ}$ \\ Stomatology Hospital, School of Stomatology, Zhejiang University School of Medicine, Clinical Research Center for Oral Diseases of \\ Zhejiang Province, Key Laboratory of Oral Biomedical Research of Zhejiang Province, Cancer Center of Zhejiang University, \\ Hangzhou 310006, China \\ Correspondence should be addressed to Jiejun Shi; sjiejun@zju.edu.cn
}

Received 23 March 2021; Revised 30 May 2021; Accepted 27 June 2021; Published 9 July 2021

Academic Editor: Dongliang Xu

Copyright ( $) 2021$ Wen Li et al. This is an open access article distributed under the Creative Commons Attribution License, which permits unrestricted use, distribution, and reproduction in any medium, provided the original work is properly cited.

\begin{abstract}
Temporomandibular joint osteoarthritis (TMJOA) is characterized by chronic inflammatory degradation of mandibular condylar cartilage (MCC). Studies have found a positive correlation between inflammation and cyclooxygenase- (COX-) 2 in OA pathology. $\mathrm{NF}-\kappa \mathrm{B}$ is a crucial transcription factor of inflammatory and immune responses in the cause of TMJOA pathology. Resveratrol (RES) plays a critical role in antioxidation and anti-inflammation. But, studies on the effects of RES on TMJOA are very limited. So, the purpose of this study is to investigate the antioxidant and protective effects of RES against MCC degradation through downregulating $\mathrm{COX}-2 / \mathrm{NF}-\kappa \mathrm{B}$ expression. In vitro studies, the MCC cells were divided into three groups: the NC group, OA group, and RES group. The optimum dose of RES $(10 \mu \mathrm{M})$ was determined. The TMJOA model of mice was created by injection of collagenase. And mice were injected with RES $(100 \mu \mathrm{g} / 10 \mu \mathrm{l}) 3$ times one week for 4 weeks in the RES group. The expressions of COX-2, P65, MMP1, MMP13, COL2, and ACAN were measured by RT-PCR. Morphological changes of MCC were studied with HE staining. The results showed that inflammation could induce MCC degradation in vitro and vivo, while RES could reverse the degradation. Meanwhile, RES could downregulate COX-2/NF- $\kappa \mathrm{B} / \mathrm{MMP}$ expression and increase cartilage markers in vitro and vivo studies. The results indicated that RES treatment had antioxidant effects against chondrocyte apoptosis by downregulating the COX-2/NF- $\kappa$ B pathway in created TMJOA.
\end{abstract}

\section{Introduction}

Temporomandibular joint osteoarthritis (TMJOA) is characterized by chronic inflammatory degradation of mandibular condylar cartilage (MCC) [1, 2]. The exact etiology of TMJOA is still unknown, but several risk factors have been reported, such as oxidative stress and inflammation [3-5]. Many studies reported that inflammatory pathway as a "triggering factor" is closely related to TMJOA [6]. Our previous study showed that interleukin- $1 \beta$ (IL- $1 \beta$ ) contributed to the pathogenesis of TMJOA and induced the inflammation and destruction of the MCC [4]. The nuclear factor kappa B $(\mathrm{NF}-\kappa \mathrm{B})$ family of transcription factors is a key regulator of immune development, immune responses, and inflammation $[7,8]$. The association between NF- $\kappa$ B and the pathogenesis of TMJOA has been confirmed in animal experiments [4]. IL- $1 \beta$-activated NF- $\kappa \mathrm{B}$ promotes OA development via its action on MCC. Therefore, in order to alleviate or even cure TMJOA, it is of great significance to understand the molecular mechanism of the effect of inflammation on TMJOA.

Cyclooxygenase (COX) is the rate-limiting enzyme for arachidonic acid to synthesize prostaglandin E2 (PGE2) - one of the major mediators involved in the degradation of articular cartilage in OA [9]. There are two isoforms of COX in human that have been described, COX-1 and COX-2.COX-1 is expressed constitutively in various tissues for homeostasis maintenance, while COX-2 is induced by numerous stimuli including excessive mechanical stress, chemical stimuli, and inflammation, being regarded as a pathological enzyme $[10,11]$. Studies have found a positive 
TABLE 1: The primer sequences for RT-PCR.

\begin{tabular}{|c|c|c|}
\hline Gene & Sequences $5^{\prime}-3^{\prime}$ & PrimerBank ID \\
\hline GAPDH & $\begin{array}{c}\text { Forward: AGGTCGGTGTGAACGGATTTG } \\
\text { Reverse: GGGGTCGTTGATGGCAACA }\end{array}$ & $126012538 \mathrm{c} 1$ \\
\hline $\mathrm{COX}-2$ & $\begin{array}{l}\text { Forward: TTCCAATCCATGTCAAAACCGT } \\
\text { Reverse: AGTCCGGGTACAGTCACACTT }\end{array}$ & $118130137 \mathrm{c} 1$ \\
\hline P65 & $\begin{array}{l}\text { Forward: GGGCTTGGAAATAGAGACATTGA } \\
\text { Reverse: GTTCACGGATGACCTCTTTGTTT }\end{array}$ & 20379991a1 \\
\hline MMP1 & $\begin{array}{c}\text { Forward: CCTTGATGAGACGTGGACCAA } \\
\text { Reverse: ATGTGGTGTTGTTGCACCTGT }\end{array}$ & $133778986 \mathrm{c} 1$ \\
\hline MMP13 & $\begin{array}{l}\text { Forward: TGTTTGCAGAGCACTACTTGAA } \\
\text { Reverse: CAGTCACCTCTAAGCCAAAGAAA }\end{array}$ & $291463259 \mathrm{c} 1$ \\
\hline ACAN & $\begin{array}{l}\text { Forward: GTGGAGCCGTGTTTCCAAG } \\
\text { Reverse: AGATGCTGTTGACTCGAACCT }\end{array}$ & $116875857 \mathrm{c} 1$ \\
\hline COL2a & $\begin{array}{l}\text { Forward: GGGTCACAGAGGTTACCCAG } \\
\text { Reverse: ACCAGGGGAACCACTCTCAC }\end{array}$ & $166064039 \mathrm{c} 1$ \\
\hline
\end{tabular}

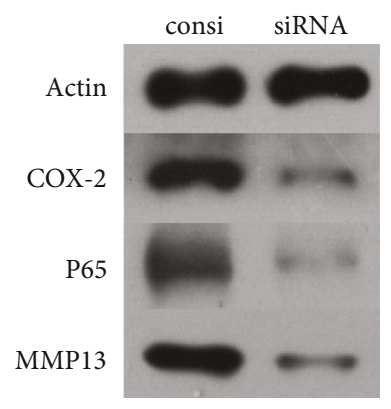

(a)

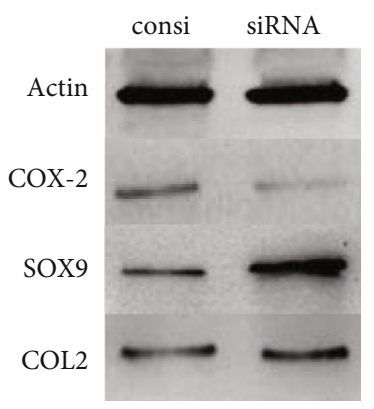

(b)

FIgURE 1: Effect of COX-2 on IL-1 $\beta$-induced chondrocyte degradation. Chondrocytes treated with COX-2 siRNA or control vehicle siRNA were subjected to IL-1 $\beta$. Whole-cell lysis was subjected to western blot analysis. (a) The effects of COX-2 on inflammation pathway. Inhibition of COX-2 could decrease the expression of P65 and MMP13. (b) The effects of COX-2 on MCC degradation. Inhibition of COX-2 could increase the expression of SOX9 and COL2.

correlation between inflammation, oxidative stress reaction, and the expression of COX-2. Induced COX-2 expression leads to the secretion of proinflammatory cytokines such as PGE2, PGH2, and VEGF, inducing the generation of oxidative stress-associated products like oxygen radicals and ROS, and resulting in the cellular injury [12, 13]. And in TMJ, COX-2 plays an important part in condylar cartilage degeneration [14]. Therefore, targeting COX-2 may be a promising method to suppress TMJOA.

Resveratrol (RES) is a kind of multifunctional biological polyphenol and plays a critical role in the cell viability, proliferation, anti-inflammation, and antioxidant [15] properties, moreover, in the prevention and progression of chronic diseases related to inflammation [16]. Some studies have demonstrated that RES alleviates rheumatoid arthritis by reducing inflammation, inhibiting MAPK signaling pathways, and suppressing angiogenesis [17]. Although some studies revealed that the intra-articular RES treatment could exert a curative effect by preventing the inflammation and cartilage destruction of TMJOA [18], the mechanism remained unknown.

In this study, we investigated the therapeutic effect and the possible mechanism of RES on MCC in vitro and in $\mathrm{OA}$ mice, then presumed that RES has restorative effects on cartilage destruction by inhibition of $\mathrm{COX}-2 / \mathrm{NF}-\kappa \mathrm{B}$ signaling pathways.

\section{Materials and Methods}

2.1. Cell Culture and RES Treatment. The procedures for cell culture were referred to Izawa's study [4]. Briefly, cells were isolated from condylar cartilage of 6-week-old female C57BL/6J mice by mechanical dissection and digestion with trypsin and collagenase II. All cells were cultured in $\mathrm{CO}_{2}$ incubator (DMEM, $37^{\circ} \mathrm{C}, 5 \% \mathrm{CO}_{2}$ ). Third cultures were treated with $5,10,20,50$, and $100 \mu \mathrm{M}$ RES (Sigma, USA) for $12 \mathrm{~h}$ and then tested by MTT to observe the optimum dose of RES on cell viability.

2.2. IL-1 $\beta$-Induced Apoptosis. The third-generation cells were divided into three groups: the normal control group (NC), osteoarthritis group (OA), and RES treatment group (RES). The OA group cells were induced by IL- $\beta$ ( $10 \mathrm{ng} / \mathrm{ml}$, PeproTech Inc., USA) [19]. The RES group cells were treated with IL-1 $\beta(10 \mathrm{ng} / \mathrm{ml})$ and RES $(10 \mu \mathrm{M})$. Cell apoptosis was measured by flow cytometry using AnnexinV Apoptosis Detection Kit I (BD Bioscience). 


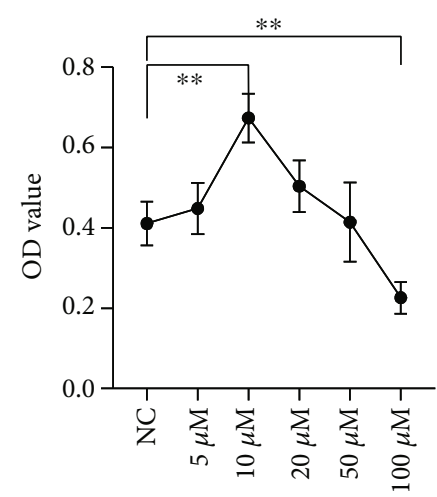

(a)

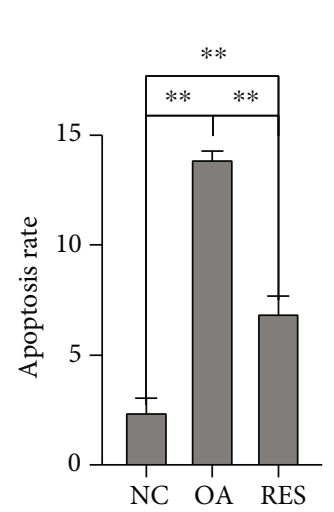

(c)
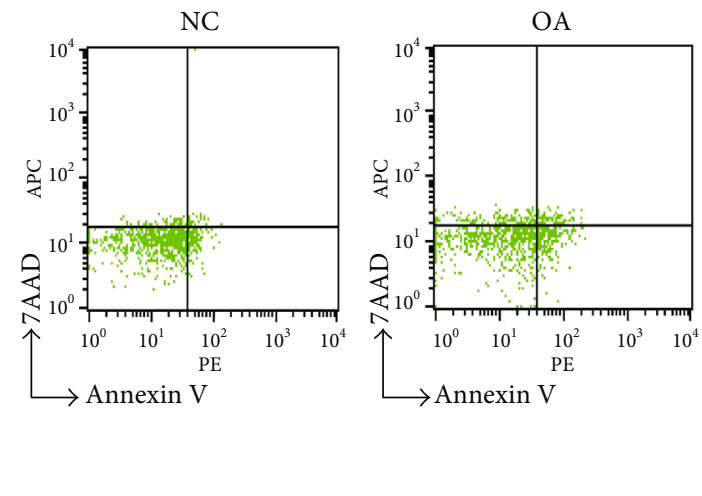

(b)

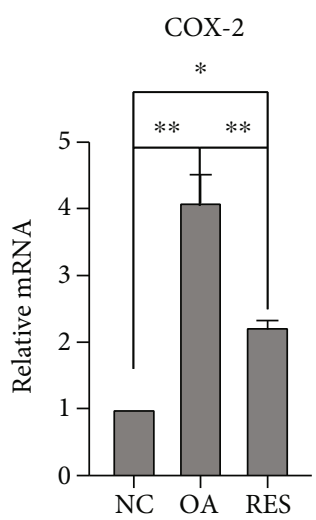

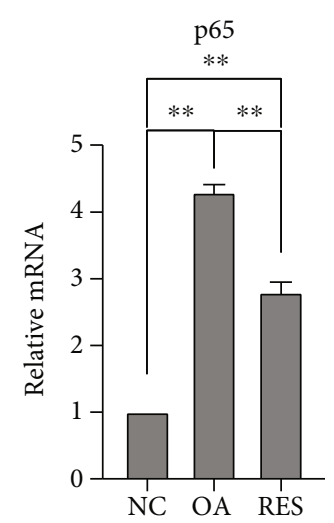

(d)

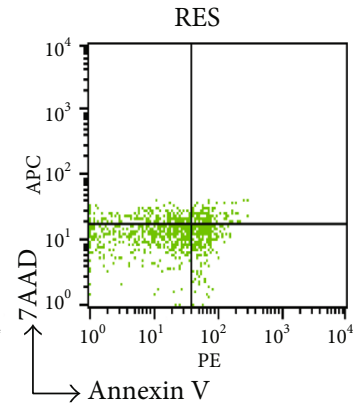

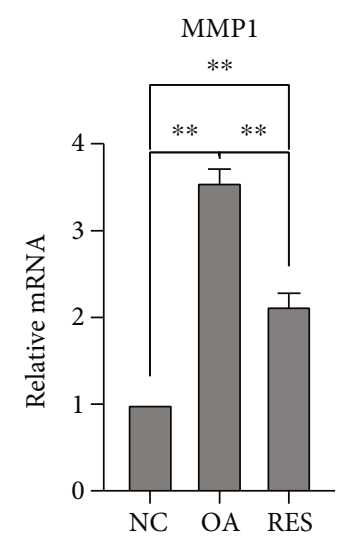

MMP13

ACAN

COL2

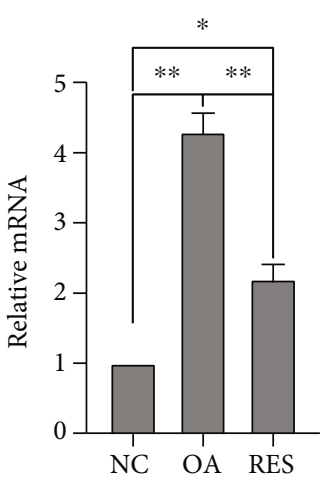

(e)
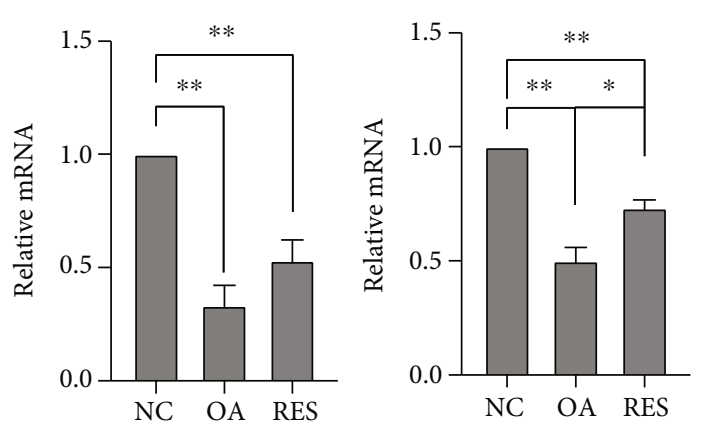

(f)

FIGURE 2: Effect of RES on IL-1 $\beta$-induced apoptosis in the chondrocytes. (a) The optimum dose of RES was determined by MTT after cotreatment for $12 \mathrm{~h}$. (b, c) Flow cytometric analysis for chondrocyte apoptosis. RES treatment could decrease cell apoptosis. (d) The mRNA expression of COX-2 and P65 in each group; RES treatment could suppress COX-2 and P65 expression in vitro. (e) The mRNA expression of MMP1 and MMP13 in each group. (f) The levels of cartilage markers' (ACAN, COL2) gene expressions in chondrocytes were determined by RT-PCR. RES treatment could increase cartilage markers' expression $\left(n=3 ;{ }^{*} P<0.05,{ }^{* *} P<0.01\right)$.

2.3. siRNA Treatment for COX-2. The cells $\left(2.0 \times 10^{5} /\right.$ well $)$ were seeded and cultured in a 6 -well culture plate until $70 \%$ confluence, the culture medium was changed to reduced serum medium (Opti-MEM, Gibco) for overnight, and then, the cells were treated with COX-2 siRNA (GenePharma, China), or control vehicle siRNA with lipofectamine reagent (Invitrogen) for $24 \mathrm{~h}$, according to the manufacturer's instructions. Then, treated cells were cultured with IL-1 $\beta$
(10 ng/ml). $24 \mathrm{~h}$ later, the cells were harvested and used for western blot analysis.

2.4. Setup TMJOA Model. 12 female mice (8 week old, 17.3 $\pm 1.5 \mathrm{~g}$ ) were purchased from the Animal Center of Zhejiang Academy of Medical Sciences. The mice were divided into the normal control group (NC), OA group (OA), and RES group (RES). The TMJOA model was set up by injection of 


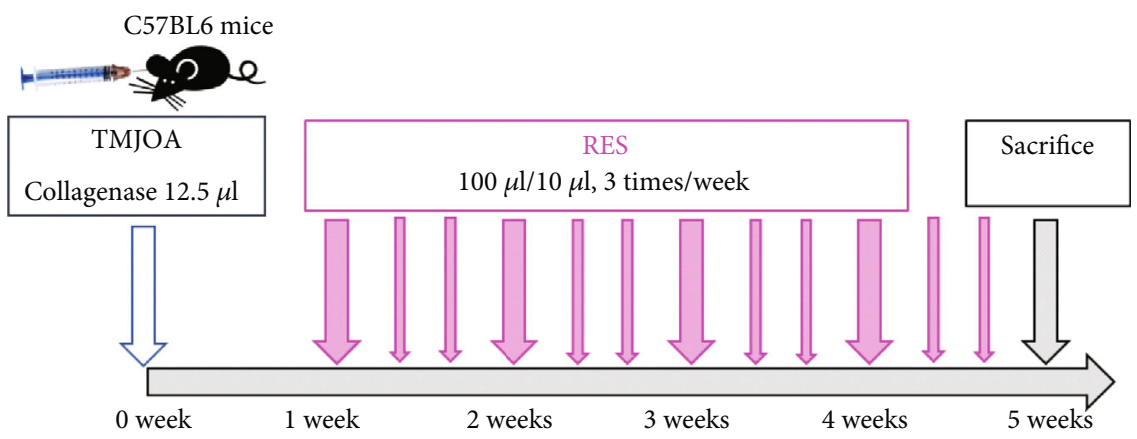

(a)
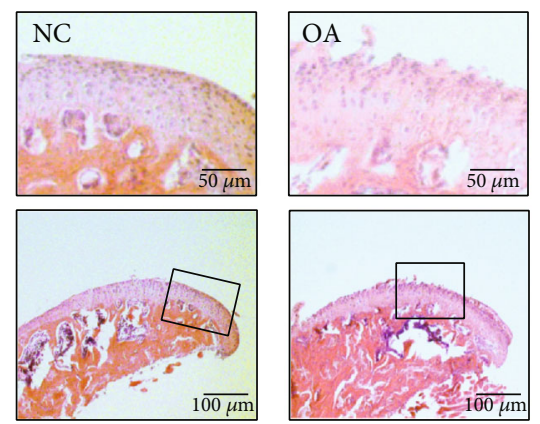

(b)

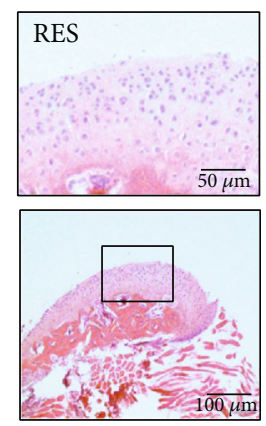

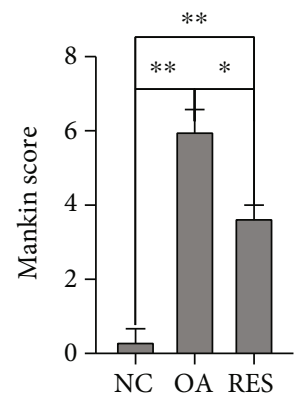

(c)

FIGURE 3: RES alleviated structure changes in mouse TMJOA model. (a) Procedure for setting up the TMJOA model and injecting RES. (b) Histological analysis of condylar cartilage by H\&E staining. RES treatment could partly reverse cartilage degradation. (c) Mankin score of the condylar articular cartilage of three groups $\left(n=3 ;{ }^{*} P<0.05,{ }^{* *} P<0.01\right)$.

collagenase $(12.5 \mu \mathrm{l}$, Sigma Biochemical, St. Louis) in the upper cavity of TMJ according to previous studies and made some modifications $[2,18]$. 1 week later, the OA mice were injected by RES (100 $\mu \mathrm{g} / 10 \mu \mathrm{l})$ [18] 3 times one week. 4 weeks later, the mice were sacrificed and the condyle was harvested. Animals were treated according to the Guidelines for Animal Care at Zhejiang University School of Medicine at Hangzhou.

2.5. Histopathology. The condylar tissues were fixed in $4 \%$ PFA, then decalcified in 10\% EDTA for 4 weeks. After dehydration, the samples were embedded in paraffin. For histological analysis, sections were deparaffinized and stained with hematoxylin and eosin (H\&E). Mankin score was used to evaluate the structure changes of condylar articular cartilage.

2.6. Measurement of COX-2/P65/MMPs and Cartilage Markers. Total RNA was extracted with TRIzol (Invitrogen), and the cDNA was synthesized according to the GeneAmp PCR kit (ABI, USA). RT-PCR was performed according to the SYBR green RT-qPCR kit (TOYOBO Corporation). All PCR reactions were performed using iCycleriQTM (BioRad, Hercules, CA, USA). The cycling conditions were 10 min at $95^{\circ} \mathrm{C}$, followed by 40 cycles: denaturation at $94^{\circ} \mathrm{C}$ for $15 \mathrm{~s}$, annealing for $30 \mathrm{~s}$ at $57^{\circ} \mathrm{C}$, and extension at $72^{\circ} \mathrm{C}$ for 30 s. The primers of GAPDH, COX-2, P65, MMPs, and cartilage markers were shown in Table 1.

2.7. Statistical Analysis. SPSS 22.0 was used for the statistical analysis, and $P$ value less than 0.05 was considered signifi- cantly different. Student's $t$-test was used for comparisons between two groups.

\section{Results}

3.1. Mechanism of COX-2 on MCC Degradation. To examine the effect and mechanism of COX-2 on cartilage degradation, chondrocytes were treated with COX-2 siRNA or control vehicle siRNA before being treated with IL-1 $\beta$. P65, MMP13, and the cartilage markers (SOX9 and COL2) were measured by western blot. The present study found that COX-2 siRNA treatment could significantly decrease the expression of P65 and MMP13 and reverse the decrease of the expression of SOX9 and COL2 induced by IL-1 $\beta$ (Figure 1).

3.2. Effects of RES on IL-1 $\beta$-Induced Apoptosis. The optimum dose of RES $(10 \mu \mathrm{M})$ was determined by MTT (Figure 2(a)). IL- $1 \beta$ induced the upregulation of apoptosis compared with the NC group. Meanwhile, RES could decrease the apoptosis induced by IL-1 $\beta$ (Figures $2(\mathrm{~b})$ and $2(\mathrm{c})$ ). The mRNA expression of COX-2, P65, and MMPs in the OA group increased significantly, and RES treatment reversed this increase (Figures 2(d) and 2(e)). The mRNA level of cartilage markers (COL2 and ACAN) decreased obviously in the OA group, and RES could increase the markers expression (Figure 2(f)).

3.3. Structure Changes of Condylar Articular Cartilage. HE staining showed normal morphology of joints in the normal control group, whereas the joints in the OA groups exhibited irregular surface. With intra-articular injection of RES, the 

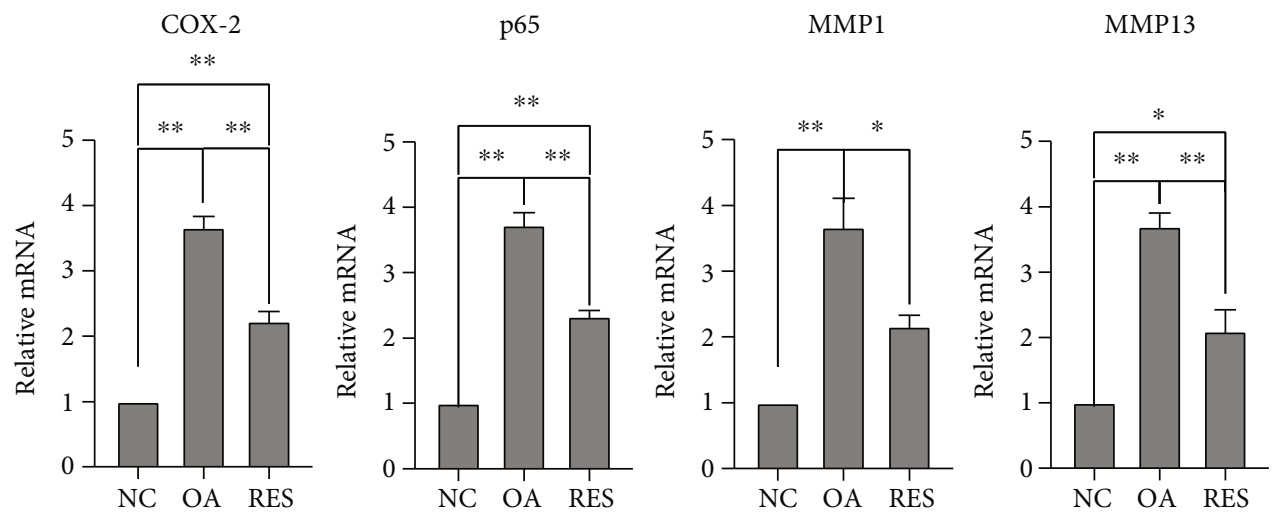

(a)
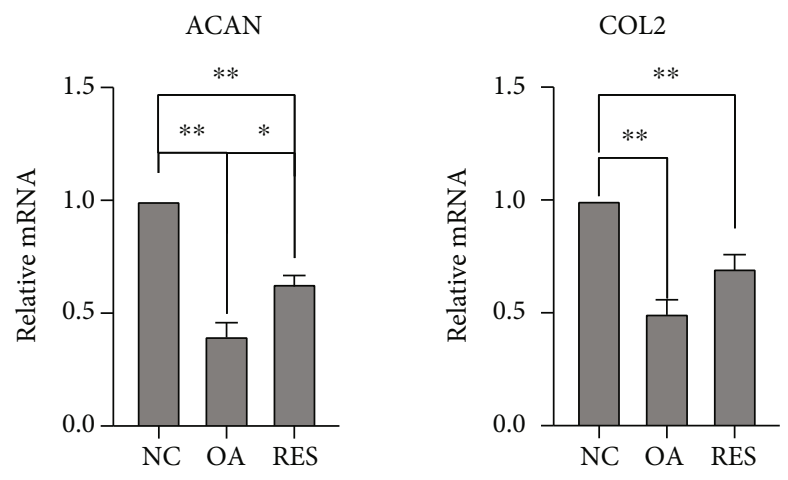

(b)

FIgURE 4: RES influenced the expression of COX-2/P65/MMPs and cartilage markers in mouse TMJOA model. (a) The mRNA expression of COX-2, P65, and MMP1 and MMP13 in MCC was detected by RT-PCR. RES treatment could suppress COX-2 expression and related inflammation pathway. (b) The levels of ACAN and COL2 gene expressions in the mouse condylar cartilage were determined by RT-PCR. RES treatment had therapeutic effects against MCC degradation $\left(n=3 ;{ }^{*} P<0.05,{ }^{* *} P<0.01\right)$.

surface of the articular cartilage was a little bit smoother than the OA group, and cartilage thickness was increased (Figure 3(b)). Mankin score revealed that RES could reverse the damage of MCC of OA mice to some extent (Figure 3(c)).

3.4. The Expression of COX-2/P65/MMPs and Cartilage Markers in MCC. Compared with the NC group, the mRNA expression of COX-2, P65, and MMP1 and MMP13 increased significantly in OA mice, while the expressions of aggrecan and COL2 were significantly decreased in TMJOA mice. RES treatment significantly upregulated the gene expressions of cartilage markers and reduced the gene expressions of COX-2, P65, and MMPs (Figure 4).

\section{Discussion}

TMJOA is mainly characterized by the release of inflammatory cytokines, such as IL- 1 and TNF- $\alpha$, leading to the destruction of cartilage matrix. COX-2 is an important proinflammatory enzyme, whose abnormal expression is a significant marker of OA in joints $[9,14]$. It is reported that normal human articular chondrocytes do not express measurable COX-2 mRNA, but inflammatory cytokines like IL- 1 and TNF- $\alpha$ can induce articular chondrocytes expressing COX-2 mRNA [20, 21]. In TMJ, COX-2 plays an important part in condylar cartilage degeneration and progression of inflammation $[5,22]$. When articular chondrocytes and synoviocytes from TMJ were treated with exogenous IL- $1 \beta$, the expression levels of COX-2/PGE2 were enhanced $[23,24]$. On the other hand, COX inhibitors decreased the expression of COX-2 and PGE2 production in condylar chondrocytes and fibroblast-like synoviocytes from TMJ, showing the anti-inflammatory effect of COX inhibitors $[21,22]$. The present study found that IL-1 could increase the protein expression of COX-2 and decrease the expression of cartilage markers, while inhibition of COX-2 by siRNA could suppress the NF- $\kappa \mathrm{B}$ pathway by descending the expression of P65 and downstream factor MMP13. Meanwhile, inhibition of COX-2 could increase the expression of cartilage markers (SOX9 and COL2), which indicated that the COX-2 may be a potential therapeutic target against MCC degradation.

RES, which is known to have anti-inflammation and antioxidation effects, plays an important role in treating OA disease. And our study verified the therapeutic effect of RES on MCC cells by flow cytometry, which demonstrated that RES significantly reversed IL- $1 \beta$-induced apoptosis in the chondrocytes. Some studies verified that RES exerted protective effects on OA through its anti-inflammatory property by the NF- $\kappa$ B pathway $[25,26]$. The present study agreed with previous studies, which revealed the anti-inflammation of RES on TMJOA by inhibiting the COX-2 gene expression, $\mathrm{NF}-\kappa \mathrm{B}$ pathway, and downstream factors MMPs. MMPs are the key enzymes related to cartilage degradation in OA. 
The proteolytic cleavage of collagens and proteoglycans by MMPs are responsible for temporomandibular disorders $[27,28]$. The present study has implicated that RES could inhibit MMP1 and MMP13 in MCC and increase the expression of cartilage markers ACAN and COL2 in vitro, which is one of the mechanism responsible for RES therapeutic effect.

In TMJOA mice, the structure of the condylar articular cartilage was destroyed significantly by injection in the upper cavity of TMJ compared to control mice. After RES treatment, histological evaluation of the cartilage tissue revealed a significantly reduced cartilage destruction compared to the OA group, which indicated the protective effects of resveratrol against MCC. Many in vivo studies demonstrated that RES may exert an antiosteoarthritic effect by inhibiting COX-2 gene expression and enzyme activity [29]. It was reported that RES decreased AGEs-stimulated expressions of MMP13 and prevented AGEs-mediated destruction of collagen II [30]. In TMJOA mice, our study found that RES could suppress the expression of COX-2, NF- $\kappa \mathrm{B}$ pathway, and MMPs and increase the expression of cartilage markers ACAN and COL2, which indicated that RES may be a promising agent in the treatment for TMJOA.

However, it should be realized that there are a few limitations of the experiments. More biological effects such as cell proliferation, more inflammatory mediators, and the mechanism through which COX-2 induced by inflammation were not demonstrated.

\section{Conclusions}

In conclusion, this study indicates the involvement of COX-2 on inflammation-induced condylar cartilage degeneration in temporomandibular osteoarthritis. The most intriguing aspect of this study is that the RES recovering MCC injury may be related to the inhibition of the expression of COX$2 / \mathrm{NF}-\kappa \mathrm{B}$. In addition, RES could increase the expressions of chondrogenic markers, suggesting that RES plays an important role in the remodeling of the cartilage in TMJOA.

\section{Data Availability}

Data used to support the findings of this study are available from the corresponding author upon request.

\section{Conflicts of Interest}

The authors declare that there are no conflicts of interest regarding the publication of this paper.

\section{Authors' Contributions}

Wen Li and Shiyu Hu contributed equally to this work.

\section{Acknowledgments}

The authors are grateful to the Zhejiang Academy of Medical Sciences for biotechnical assistance. This work was financially supported by the Natural Science Foundation of Zhejiang Province (LY18H140001) and Zhejiang public welfare Technology Application Research Plan (LGF21H140003).

\section{References}

[1] G. A. Zarb and G. E. Carlsson, "Temporomandibular disorders: osteoarthritis," Journal of Orofacial Pain, vol. 13, no. 4, pp. 295-306, 1999.

[2] W. Li, M. Wu, S. Jiang, W. Ding, Q. Luo, and J. Shi, "Expression of ADAMTs-5 and TIMP-3 in the condylar cartilage of rats induced by experimentally created osteoarthritis," Archives of oral biology, vol. 59, no. 5, pp. 524-529, 2014.

[3] A. S. Marchev, P. A. Dimitrova, A. J. Burns, R. V. Kostov, A. T. Dinkova-Kostova, and M. I. Georgiev, "Oxidative stress and chronic inflammation in osteoarthritis: can NRF2 counteract these partners in crime?," Annals of the New York Academy of Sciences, vol. 1401, no. 1, pp. 114-135, 2017.

[4] T. Izawa, H. Mori, T. Shinohara et al., "Rebamipide attenuates mandibular condylar degeneration in a murine model of TMJOA by mediating a chondroprotective effect and by downregulating RANKL-mediated osteoclastogenesis," PLoS One, vol. 11, no. 4, article e0154107, 2016.

[5] C. Jiang, P. Luo, X. Li, P. Liu, Y. Li, and J. Xu, "Nrf2/ARE is a key pathway for curcumin-mediated protection of TMJ chondrocytes from oxidative stress and inflammation," Cell Stress \& Chaperones, vol. 25, no. 3, pp. 395-406, 2020.

[6] E. B. P. Lopes, A. Filiberti, S. A. Husain, and M. B. Humphrey, "Immune contributions to osteoarthritis," Current osteoporosis reports, vol. 15, no. 6, pp. 593-600, 2017.

[7] S. Mitchell, J. Vargas, and A. Hoffmann, "Signaling via the $\mathrm{NF} \kappa \mathrm{B}$ system," Wiley Interdisciplinary Reviews: Systems Biology and Medicine, vol. 8, no. 3, pp. 227-241, 2016.

[8] M. C. Choi, J. Jo, J. Park, H. K. Kang, and Y. Park, "NF-B signaling pathways in osteoarthritic cartilage destruction," Cells, vol. 8, no. 7, p. $734,2019$.

[9] P. Alstergren and S. Kopp, "Prostaglandin E2 in temporomandibular joint synovial fluid and its relation to pain and inflammatory disorders," Journal of Oral \& Maxillofacial Surgery, vol. 58, no. 2, pp. 180-186, 2000.

[10] B. Lyons-Giordano, M. A. Pratta, W. Galbraith, G. L. Davis, and E. C. Arner, "Interleukin-1 differentially modulates chondrocyte expression of cyclooxygenase-2 and phospholipase A2," Experimental Cell Research, vol. 206, no. 1, pp. 58-62, 1993.

[11] S. C. Su, K. Tanimoto, Y. Tanne et al., "Celecoxib exerts protective effects on extracellular matrix metabolism of mandibular condylar chondrocytes under excessive mechanical stress," Osteoarthritis and Cartilage, vol. 22, no. 6, pp. 845-851, 2014.

[12] S. S. Barbieri, S. Eligini, M. Brambilla, E. Tremoli, and S. Colli, "Reactive oxygen species mediate cyclooxygenase- 2 induction during monocyte to macrophage differentiation: critical role of NADPH oxidase," Cardiovascular Research, vol. 60, no. 1, pp. 187-197, 2003.

[13] L. Qiu, Y. Zhang, D. C. Do et al., "miR-155 modulates cockroach allergen- and oxidative stress-induced cyclooxygenase-2 in asthma," The Journal of Immunology, vol. 201, no. 3, pp. 916-929, 2018.

[14] H. W. Fan, G. Y. Liu, C. F. Zhao, X. F. Li, and X. Y. Yang, "Differential expression of COX-2 in osteoarthritis and rheumatoid arthritis," Genetics and Molecular Research, vol. 14, no. 4, pp. 12872-12879, 2015.

[15] W. Wang, L. Sun, P. Zhang, J. Song, and W. Liu, "An antiinflammatorycell-free collagen/resveratrol scaffold for repairing osteochondral defects in rabbits," Acta biomaterialia, vol. 10, no. 12, pp. 4983-4995, 2014. 
[16] L. Malaguarnera, "Influence of resveratrol on the immune response," Nutrients, vol. 11, no. 5, p. 946, 2019.

[17] G. Yang, C. C. Chang, Y. Yang et al., "Resveratrol alleviates rheumatoid arthritis via reducing ROS and inflammation, inhibiting MAPK signaling pathways, and suppressing angiogenesis," Journal of Agricultural and Food Chemistry, vol. 66, no. 49, pp. 12953-12960, 2018.

[18] P. Yuce, H. Hosgor, S. F. Rencber, and Y. Yazir, "Effects of intra-articular resveratrol injections on cartilage destruction and synovial inflammation in experimental temporomandibular joint osteoarthritis," Journal of Oral and Maxillofacial Surgery, vol. 79, no. 2, pp. 344.e1-344.e12, 2021.

[19] N. Rasheed, A. Alghasham, and Z. Rasheed, "Lactoferrin from Camelus dromedarius inhibits nuclear transcription factorkappa B activation, cyclooxygenase- 2 expression and prostaglandin E2 production in stimulated human chondrocytes," Pharmacognosy research, vol. 8, no. 2, pp. 135-141, 2016.

[20] X. Li, P. He, Y. Hou et al., "Berberine inhibits the interleukin1beta-induced inflammatory response via MAPK downregulation in rat articular chondrocytes," Drug development research, vol. 80, no. 5, pp. 637-645, 2019.

[21] R. Y. Au, T. K. Al-Talib, A. Y. Au, P. V. Phan, and C. G. Frondoza, "Avocado soybean unsaponifiables (ASU) suppress TNF-alpha, IL-1beta, COX-2, iNOS gene expression, and prostaglandin E2 and nitric oxide production in articular chondrocytes and monocyte/macrophages," Osteoarthritis and Cartilage, vol. 15, no. 11, pp. 1249-1255, 2007.

[22] N. Ouyang, Y. Zhao, Q. Chen et al., "The effect of celecoxib in traumatic heterotopic ossification around temporomandibular joint in mice," Osteoarthritis and Cartilage, vol. 28, no. 4, pp. 502-515, 2020.

[23] K. Tanimoto, Y. Iwabuchi, Y. Tanne et al., "Interleukin-1 beta affects cyclooxygenase-2 expression and cartilage metabolism in mandibular condyle," Archives of Oral Biology, vol. 56, no. 11, pp. 1412-1418, 2011.

[24] K. Satoh, N. Ogura, M. Akutsu et al., "Expression of cyclooxygenase- 1 and -2 in IL- $1 \beta$-induced synovitis of the temporomandibular joint," Journal of Oral Pathology \& Medicine, vol. 38, no. 7, pp. 584-590, 2009.

[25] X. Xu, X. Liu, Y. Yang et al., "Resveratrol Exerts AntiOsteoarthritic Effect by Inhibiting TLR4/NF- $\kappa$ B Signaling Pathway via the TLR4/Akt/FoxO1 Axis in IL- $1 \beta$-Stimulated SW1353 Cells," Drug Design, Development and Therapy, vol. 14, pp. 2079-2090, 2020.

[26] H. Yi, W. Zhang, Z. M. Cui et al., "Resveratrol alleviates the interleukin- $1 \beta$-induced chondrocytes injury through the NF$\kappa \mathrm{B}$ signaling pathway," Journal of Orthopaedic Surgery and Research, vol. 15, no. 1, p. 424, 2020.

[27] K. Yoshida, S. Takatsuka, E. Hatada et al., "Expression of matrix metalloproteinases and aggrecanase in the synovial fluids of patients with symptomatic temporomandibular disorders," Oral Pathology, Oral Radiology, and Endodontology, vol. 102, no. 1, pp. 22-27, 2006.

[28] Y. Asakawa-Tanne, S. Su, R. Kunimatsu et al., "Effects of enzymatic degradation after loading in temporomandibular joint," Journal of Dental Research, vol. 94, no. 2, pp. 337343, 2015.
[29] N. Elmali, I. Esenkaya, A. Harma, K. Ertem, Y. Turkoz, and B. Mizrak, "Effect of resveratrol in experimental osteoarthritis in rabbits," Inflammation Research, vol. 54, no. 4, pp. 158-162, 2005.

[30] F. C. Liu, L. F. Hung, W. L. Wu et al., "Chondroprotective effects and mechanisms of resveratrol in advanced glycation end products-stimulated chondrocytes," Arthritis research \& therapy, vol. 12, no. 5, p. R167, 2010. 